\title{
Zero reference signal for displacement measuring systems by use of speckle
}

\author{
Fernando Pérez-Quintián, José Alonso, Jesús Atencia, and Eusebio Bernabeu
}

\begin{abstract}
An optical method for generating a reference signal for an incremental displacement measurement system is proposed. We achieved this zero reference signal by comparing two speckle patterns arriving from two symmetric diffusers, which are used as natural random codes that are identical only when the reading head is located equidistantly between the diffusers. The comparison of the speckles is obtained either by interference, as in a Michelson interferometer, or by intensity correlations. (c) 2003 Optical Society of America
\end{abstract}

OCIS codes: $\quad 030.6140,120.3940,120.4640$.

\section{Introduction}

A reference signal, or zero reference, is an important addition to an incremental displacement measurement system for achieving absolute position, an origin of a coordinate, or a machine home position. By an incremental displacement measurement system, also known as an encoder, we mean a displacement transducer that generates two periodic signals with a $\pm 90^{\circ}$ phase delay between them. The period of the signals determines the transducer resolution, so the displacement from one position to the other is recorded in a counter memory as a multiple of the signal period. The phase between the signals determines the direction of the motion, so signal periods are added to or subtracted from the counter memory according to the phase sign. This measurement scheme allows for complete motion control of the moving part of the system relative to the other. In the more usual encoder setup, a glass or steel grating scale is fixed on one part. A set of reading gratings is fixed to the other part and moves with

F. Pérez-Quintián (fperez@fi.uba.ar) is with the Departamento de Física, Facultad de Ingeniería, Universidad de Buenos Aires, Paseo Colón 850, (1063) Buenos Aires, Argentina. J. Alonso (j.alonso@fis.ucm.es) and E. Bernabeu are with the Departamento de Óptica, Facultad de Ciencias Físicas, Universidad Complutense, Ciudad Universitaria, (28040) Madrid, Spain. J. Atencia is with the Departamento de Física Aplicada, Facultad de Ciencias Físicas, Universidad de Zaragoza, Pedro Cerbuna, 12 (50009) Zaragoza, Spain.

Received 14 April 2003; revised manuscript received 26 June 2003.

0003-6935/03/346797-07\$15.00/0

(C) 2003 Optical Society of America respect to the scale. By interference, moiré fringes, fringe projection with spatially incoherent light, or another optical phenomenon involving two or more gratings, the two encoder signals are generated. ${ }^{1-5}$

The shape of the reference signal is that of a lone pulse with a half-height width that is equal to the signal generated by the encoder. Traditionally, this pulse is generated by the correlation of two identical binary strip codes that are printed near and aligned with the gratings on the scale and on the reading head. These codes are also called reference marks. A transparent strip on the printed pattern corresponds to a 1 in the binary code, whereas an opaque strip on the pattern corresponds to a 0 . The codes are designed such that when light passes through the strip there are few coincidences of high bits except for the position at which the two codes are perfectly matched (zero position). At this position a maximum of light reaches the photodetectors and a sharp pulse is obtained. This pulse has a triangular shape, and its width at the base is twice the width of a code strip. Thus, if the encoder's incremental signals have a period of $20 \mu \mathrm{m}$, strips of the reference marks should have the same width. They will generate triangular pulses with widths of $40 \mu \mathrm{m}$ at the base. If the electronic comparison level is locked at half of the total height, the resultant electronic signal will be of $20 \mu \mathrm{m}$. Various code design methods have been proposed to improve the contrast of the zero reference signal (which can be defined as the ratio of the total number of transparent strips to the maximum number of coincidences out of the zero position). ${ }^{6-7}$

When an encoder resolution of more than $20 \mu \mathrm{m}$ is required, the setup described above begins to fail. A 
model that compensates for the fact that geometrical light propagation from one code to the other is no longer valid and for diffraction must be constructed. For very high-resolution encoders $(10 \mu \mathrm{m}$ or less $)$, the reference-signal generation scheme described above demands extremely short distances between the grating scale and the reading window, along with an unattainable tolerance for this distance, for a reliable and repeatable reference signal to be achieved. If larger distances are employed, diffraction causes widening and loss of contrast of the correlation pulse. The narrower the strips of the code, the stronger are the diffraction effects. For that reason other optical reading schemes are needed.

In this paper we propose an optical reader based on correlation of speckle phase or intensity. The method is robust and exhibits large mounting tolerances yet allows for the generation of reference pulses narrow enough to be used in high-resolution encoders. The general principle is discussed in Section 2. The experimental setup that we propose is described in Section 3, and experimental results that demonstrate the utility of the technique are presented in Section 4.

\section{Operation Principle}

The speckle phenomenon is good candidate for implementing a zero reference because of its characteristic fine structure, which is easily ruled by geometrical parameters. When coherent light shines upon a rough surface, the mean radius $\varepsilon$ of the speckle produced on a screen placed at a distance $l$ from it is

$$
\varepsilon=\lambda(l / a)
$$

where $\lambda$ is the wavelength and $a$ is the radius of the area illuminated by the diffuser (formally, the mutual intensity of the scattered fields in the observation plane is given, up to multiplicative constants, by the Fourier transform of the intensity distribution incident upon the diffuser ${ }^{8}$ ). If the diffuser is a onedimensional rough surface, the resultant speckle will also be invariant along the corrugation direction $(y$ axis in Fig. 1). This structure can be thought of as having a random code. The usual way to obtain a signal when the code is in the correct position is to put a mask over the detector that is a positive of the desired speckle pattern. With this method there is the problem that, if the distance between the detector and the diffuser changes a little, the signal will disappear; the reason is that, whereas the mask is fixed, the speckle changes its size with $l$ according to Eq. (1). Therefore, to overcome this problem, we propose a system with two diffusers, one symmetric to the other, to produce two identical speckles such that, although the distance between the detection head and the diffusers changes, and the speckles also change, the speckles remain identical to each other. The ways in which we compared the two speckle patterns to obtain a signal is described in Section 3.

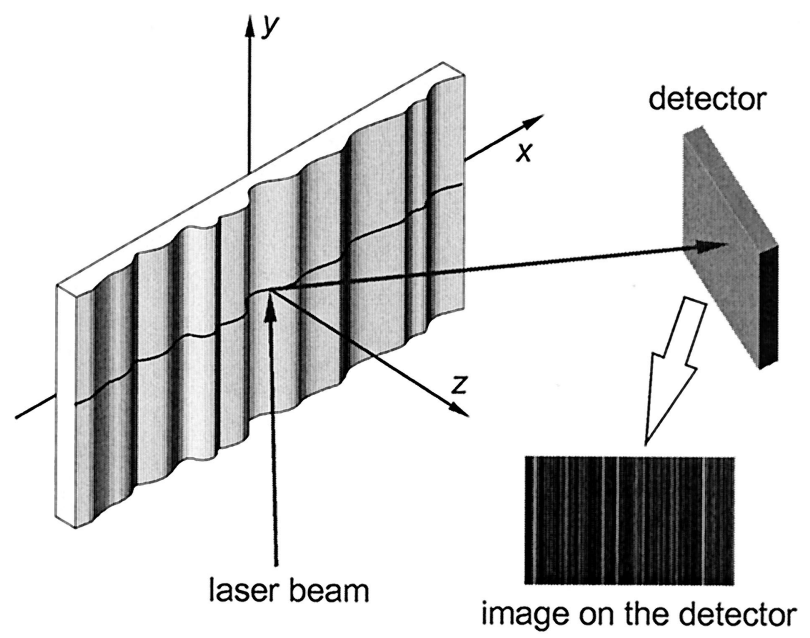

Fig. 1. Speckle pattern produced by a one-dimensional random surface.

\section{Experimental Setup}

Figure 2 shows a top view of the experimental setup that we used to test ways in which to generate a reference signal by use of speckle. A He-Ne laser beam whose divergence is ruled by lens $\mathrm{L}$ is divided by beam splitter BS and is directed by means of mir-

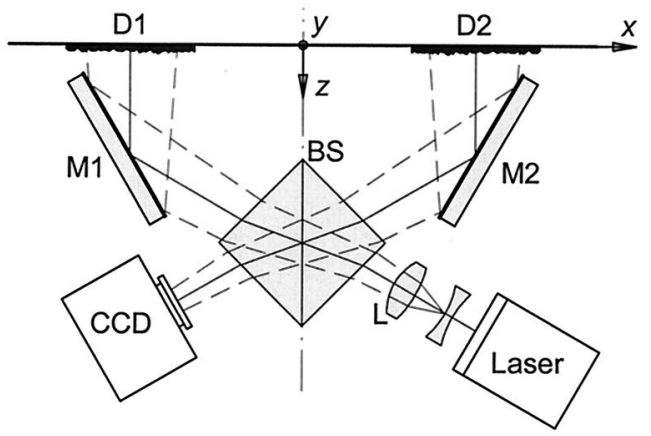

(a)

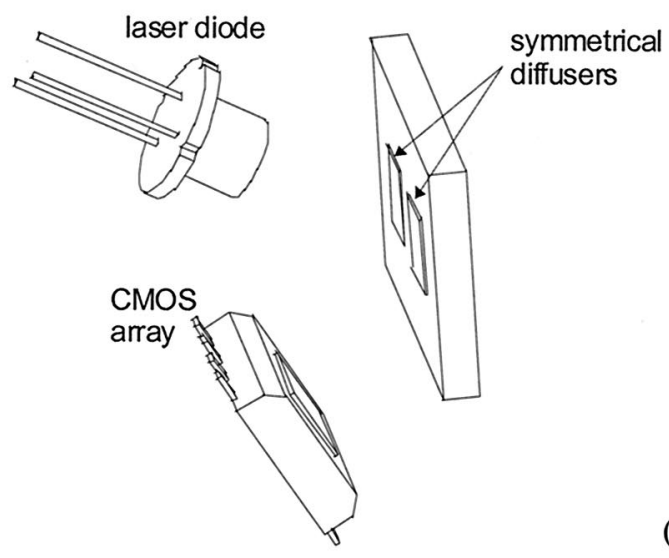

(b)

Fig. 2. (a) Top view of the experimental setup. (b) Practical implementation with enhanced-backscattering diffusers and a diode laser. 
rors $\mathrm{M} 1$ and $\mathrm{M} 2$ to symmetric diffusers $\mathrm{D} 1$ and D2. The sizes of the optics and of the diffusers are of the order of $10 \times 10 \mathrm{~mm}$. The light scattered by each diffuser, after it again is divided by the beam splitter and reflected off the mirror is recombined and detected by a CCD camera. The diffusers are mounted on to a computer-controlled motor. The total optical path between the laser and the detector is $25 \mathrm{~cm}$ long.

The system could be arranged in a simpler way to eliminate the mirrors, but then it would be necessary to have diffusers that scatter light mainly in the backward direction. Such diffusers could be constructed, for example, as a collection of facets at $45^{\circ}$ and random heights. Because we were interested above all in testing the utility of the method, we constructed the diffusers in the way described by Luna et al. ${ }^{9}$ but on top of a silver halide gelatin upon a glass plate instead of upon a photoresist, which slightly scattered light about the specular direction. We characterized the diffuser surface with an atomicforce microscope, obtaining a roughness of $0.15 \mu \mathrm{m}$ and a correlation length of $5.8 \mu \mathrm{m}$ that were consistent with the observed angular dispersion $\left(\sim 6^{\circ}\right)$.

Although most of the measurements were performed with the laboratory setup just described, a much smaller configuration was also arranged, which used a semiconductor laser without any collimating optics. Backscattered light was directly collected on a complementary metal-oxide semiconductor (CMOS) array. Because of the low backscattering efficiency of the available diffusers the signal level was much smaller than in the laboratory setup, but the remaining signal characteristics described below were identical.

The resolution of the setup depends essentially on two variables: The first one is the size of the speckle [Eq. (1)] and the second is the speckle pattern displacement for a given displacement of the diffusers. According to Yamaguchi, ${ }^{10}$ speckle pattern displacement $d$, as a function of diffuser displacement $\delta$, is given by

$$
d=\delta\left[\frac{L_{2} \cos ^{2} \theta_{1}}{L_{1} \cos ^{2} \theta_{2}}+\cos \theta_{2}\right],
$$

where $\theta_{1}$ is the incident angle with respect to the $z$ axis (Fig. 1), $\theta_{2}$ is the observation angle, $L_{1}$ is the distance between the diffuser and the point source, and $L_{2}$ is the distance between the diffuser and the observation point. So, for our setup in Fig. 2, we have $\theta_{1}=\theta_{2}=0$ and $L_{1}=L_{2}$; therefore, $d=2 \delta$. Taking into account that because of the beam splitter the speckle patterns move along opposite directions, we have a displacement amplification on the CCD of the order of four times the displacement along the $x$ axis.

\section{Experimental Results}

We made two approaches to using speckle as a mean of identifying a precise position (narrow pulse). In the first, we caused the light coming from the two diffusers interfere. As the diffusers moved with re- spect to the rest of the system, two phenomena took place: Interference fringes also moved as relative phase changes, but also the speckle pattern was correlated in phase and amplitude. These phenomena along with detector position and size determined the signal obtained. In the second approach, the intensity patterns coming the diffusers were separated and detected with arrays of detectors that spatially resolved the speckle grains and correlated the pattern without interference. In this way, only two phenomena were involved: the size of the speckle grains and the position, size, and number of detectors in the array.

\section{Interference Measurement}

The experimental setup shown in Fig. 2 is similar to that of a Michelson interferometer but with the mirrors been replaced by symmetrical diffusers. The diffusers behave as partially coherent sources (in the spatial sense), i.e., the coherence area, given by the mean transversal structure of the speckle grain, and the coherence length, given by its mean length, $\lambda$ $l^{2} / a^{2}$. The intensity on the CCD is

$$
I=I_{1}+I_{2}+\sqrt{I_{1} I_{2}} \cos \left(\phi_{12}\right),
$$

where $I_{1}$ and $I_{2}$ are the intensity patterns produced by diffusers $\mathrm{D} 1$ and D2, respectively, and $\phi_{12}$ is the phase difference between the fields. When the diffusers are centered with respect to the rest of the system (BS, M1, and M2) we observe interference fringes modulated by the speckle pattern, because phase difference $\phi_{12}$ between the fields that corresponds to each pair of speckle grains that come from each diffuser is the same. Only then is $\phi_{12}$ constant on the whole CCD surface. The shapes of the fringes depend on the exact position of the diffusers in relation to the beam splitter. In a situation with a perfect centering, and assuming that the substrate onto which the diffusers are placed is flat, there should be

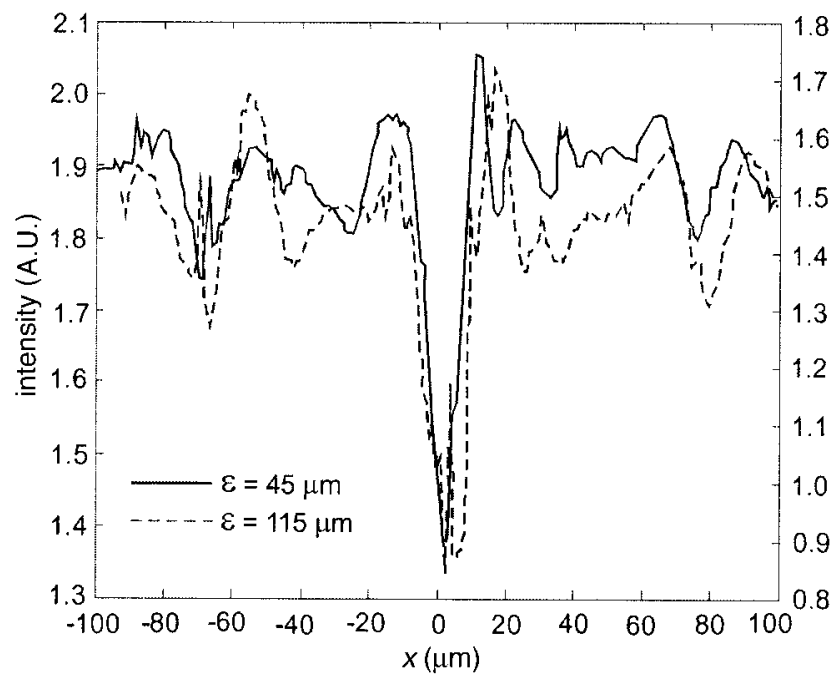

Fig. 3. Total intensity on the CCD as a function of diffuser position for the interference measurement method. The two curves correspond to two speckle sizes. 


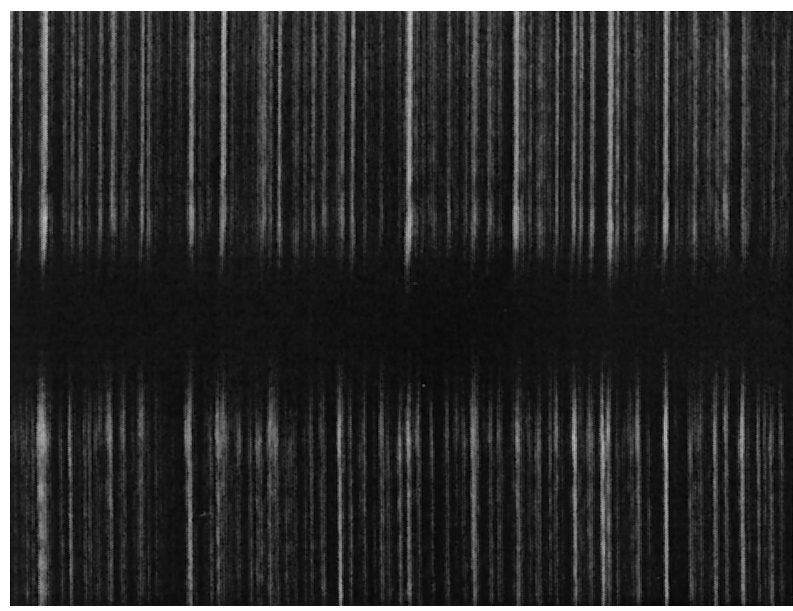

Fig. 4. Intensity pattern on the CCD with the setup chosen to calculate intensity correlations.

no fringes, merely the speckle pattern. Misalignment and lack of flatness of the diffusers generate a more-or-less regular fringe pattern. We obtain a maximum value when the path difference between the branches is a multiple of $\lambda\left(\phi_{12}=0\right)$. As the diffusers begin to move, one of the speckle patterns moves in one direction on the CCD and the other speckle pattern moves in the opposite direction, and, for this reason, the fringes begin to lose contrast. This reaction leads to a sharp drop in the signal from $\langle I\rangle=4\left\langle I_{1}\right\rangle$ toward $\langle I\rangle=2\left\langle I_{1}\right\rangle$ (we assume that $\left\langle I_{1}\right\rangle=$ $\left\langle I_{2}\right\rangle$, where \langle\rangle means the mean value taken over the position), because phase difference $\phi_{12}$ becomes random. In case the phase difference has a value $\phi_{12}=$ $\pi$ at the centered position, the signal would be $\langle I\rangle=0$, and then, as the diffusers move, the signal will rise toward $\langle I\rangle=2\left\langle I_{1}\right\rangle$. In the worst case, $\phi_{12}= \pm \pi / 2$ at the centered position, producing a signal that is, in principle, equal to $2\left\langle I_{1}\right\rangle$ in the middle but has a peak just before it and a valley after it (or vice versa).

To carry out the measurement we displace the diffusers in steps of $1 \mu \mathrm{m}$, from $-100 \mu \mathrm{m}$ to $100 \mu \mathrm{m}$ with respect to the centered position. At each step we take the image on the CCD and sum over all the pixels. Figure 3 shows the result of two of such measurements. The only difference between them is the speckle size, which is controlled by the size of the illuminated area on the diffusers, which in turns depends on the focal length of collimating lens L in Fig. 2. For the pulse shown in Fig. 3 by the solid curve, the mean size of the speckle grains was $45 \mu \mathrm{m}$, whereas it was $115 \mu \mathrm{m}$ for the pulse shown by the dashed curve. To obtain this increase in the speckle grain size in our arrangement we had to increase the distance from the diffusers to the CCD from $\sim 25 \mathrm{~mm}$ to $\sim 60 \mathrm{~mm}$. Despite the large change in distance (take into account that the tolerance in the technology based on strip-code correlation is usually less than $0.05 \mathrm{~mm}$ ), the pulse width increases just $\sim 50 \%$. For changes in the distance between diffusers and a reading head smaller than $5 \mathrm{~mm}$, we did not observe any modification of the pulse shape.

\section{Intensity Correlation Measurement}

The intensity correlation measurements are made by means of the experimental configuration of Fig. 2, with just one modification: diffuser D1 is slightly
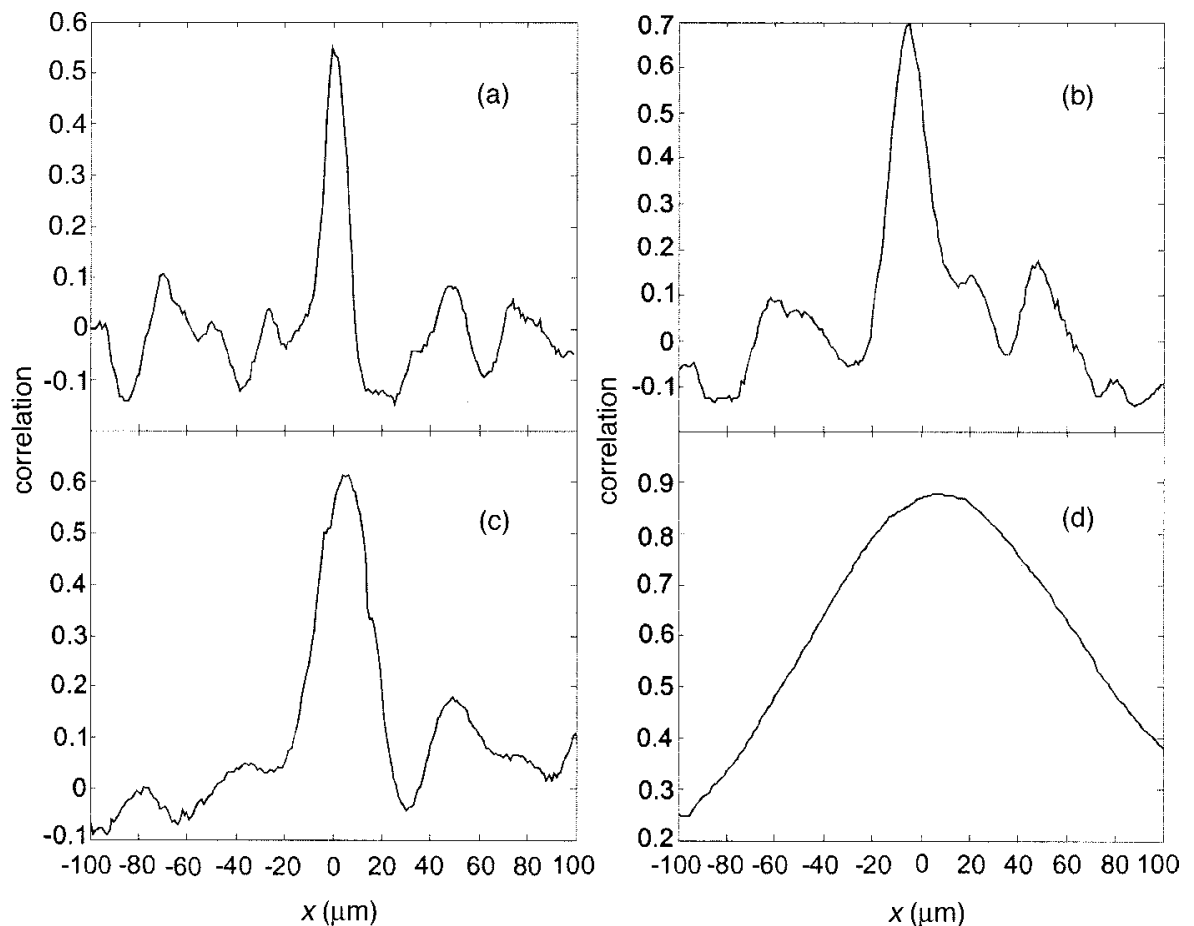

Fig. 5. Intensity correlations as a function of the symmetric diffusers' positions. Speckle size: (a) $45 \mu \mathrm{m}$, (b) $137 \mu \mathrm{m}$, (c) $116 \mu \mathrm{m}$, (d) $\sim 1 \mathrm{~mm}$. The amplification factor between diffuser displacement and speckle displacement is $\sim 2$ in (a), (c), and (d) and $\sim 3.7$ in (b). 
tilted about the $x$ axis such that its speckle pattern is now registered at the CCD array vertically displaced with respect to the speckle pattern coming from D2, as shown in Fig. 4.

The measurement method is that described in Section 5 , but here instead of summing over the pixels, we compute the correlation between two image lines of the CCD, one from to the speckle pattern at the top and the other one from the speckle pattern at the bottom.

The experimental results are shown in Fig. 5. For Figs. 5(a)-(c) the CCD was placed at the same position, such that $d \cong 2 d$ holds for all three cases. We varied the sizes of the speckle grains by changing the focal length of the collimating lens to $45 \mathrm{~mm}$ for Fig. 5(a), $116 \mathrm{~mm}$ for Fig. 5(c), and $\sim 1 \mathrm{~mm}$ for Fig. 5(d). For all cases, a variation of the distance from the reading head to the plane of the diffusers of $\pm 5 \mathrm{~mm}$ did not produce any appreciable change in the correlation signal.

For Fig. 5(b) the same lens was used as for Fig. 5(a), but the camera was placed farther from the diffusers [ $\sim 25 \mathrm{~mm}$ for Figs. 5(a), 5(c), and 5(d) to $\sim 75 \mathrm{~mm}$ for Fig. $5(\mathrm{~b})]$, so $d \cong 3.7 d$ and the size of the speckles increased to $137 \mathrm{~mm}$. Despite this increase in speckle size, the larger amplification factor of the speckle displacement caused by the larger value of L2 finally led to the narrower pulse in Fig. 5(b). This fact also demonstrates the invariance of the correlation signal with distance $z$. With collimated light, the displacement of the whole reading head (along the $z$ direction) is equivalent to a separation of the CCD from the beam splitter. As we were not using collimated light, the separation of the CCD slightly changed the width of the pulse, as can certainly be observed from Fig. 5 (b).

We did not reach maximum correlation (unity) in any of the measurements because of imperfections in the experimental setup, mainly in the diffusers. The most critical parameters are lack of symmetry, roughness along the $y$ direction, and lack of parallelism between the diffusers. Decorrelation between the speckle patterns grows exponentially with roughness and with the angle between the diffusers.

\section{Binary Correlation}

Most encoders should be able to measure displacements with a relative velocity between the moving parts of $\sim 1 \mathrm{~m} / \mathrm{s}$. In any practical implementation of the technique described above, the intensity correlation must be performed in real time, reducing the processing of acquired data to a minimum. For example, if we intend to have at least 10 samples in a $10-\mu \mathrm{m}$-wide reference pulse, we need a processing rate of $1 \mathrm{MHz}$ to cope with a velocity of $1 \mathrm{~m} / \mathrm{s}$. Signal acquisition and correlation processing should be completed in $1 \mu \mathrm{s}$. In our experiment we had to deal with two linear arrays of intensity values of the speckle patterns, $\left\{I_{i}\right\}_{i=1 \ldots N}$, one from D1 and the other from D2. To simplify postprocessing we performed binary correlation of the two arrays. In this section we investigate how binarization affects the quality of the reference signal.
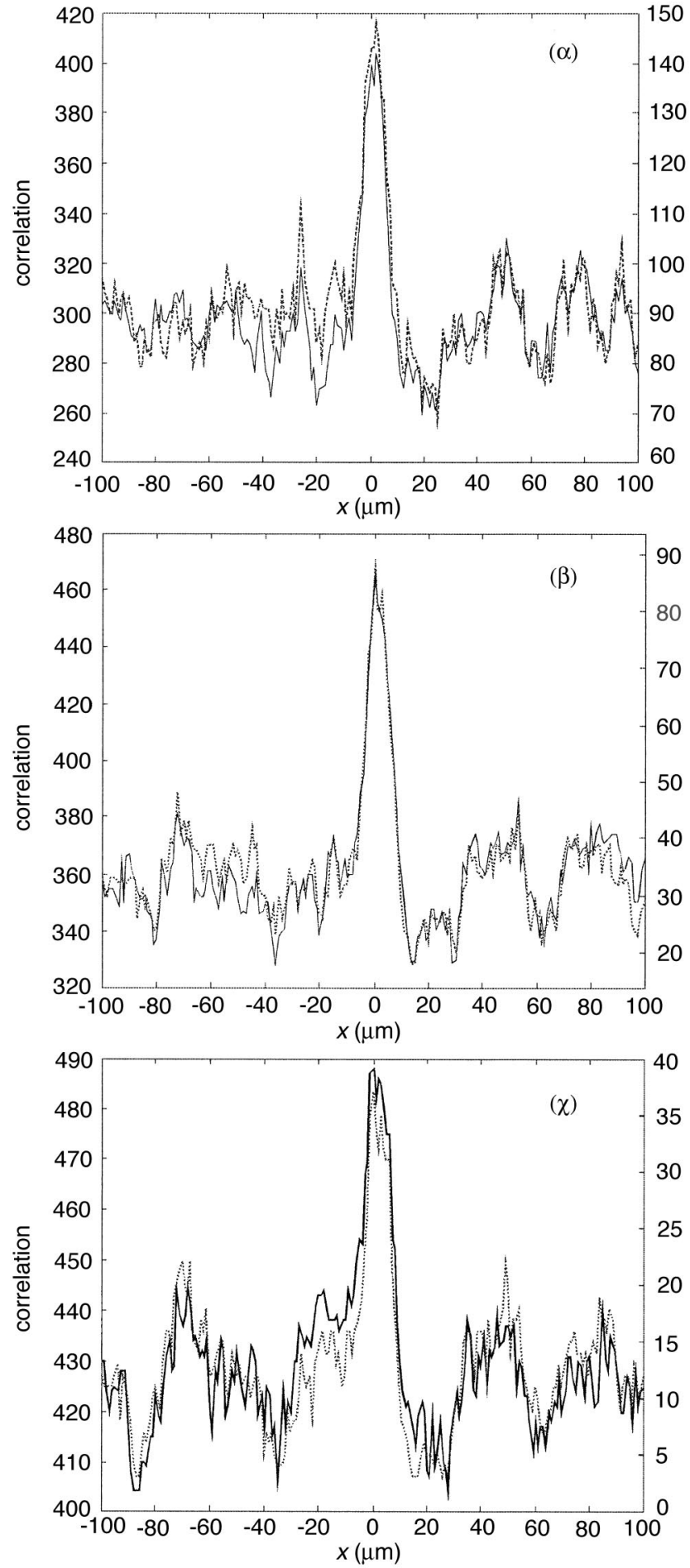

Fig. 6. Effect of intensity binarization on the correlation signal presented in Fig. 5(c). Three different thresholds were chosen. (a) Mean intensity, (b) mean intensity plus half of the variance of the intensity, (c) mean intensity plus variance of the intensity. Pulses obtained with expression (4) are shown with solid curves; with expression (5), with dashed curves.

We chose the setup parameters that correspond to the pulse shown in Fig. 5(c). Each bit of binarized code $J_{i}$ was obtained as the logic value of the expression $I_{i}>I_{T}$, where $I_{T}$ is the threshold. Let us call $\sigma_{l}$ 

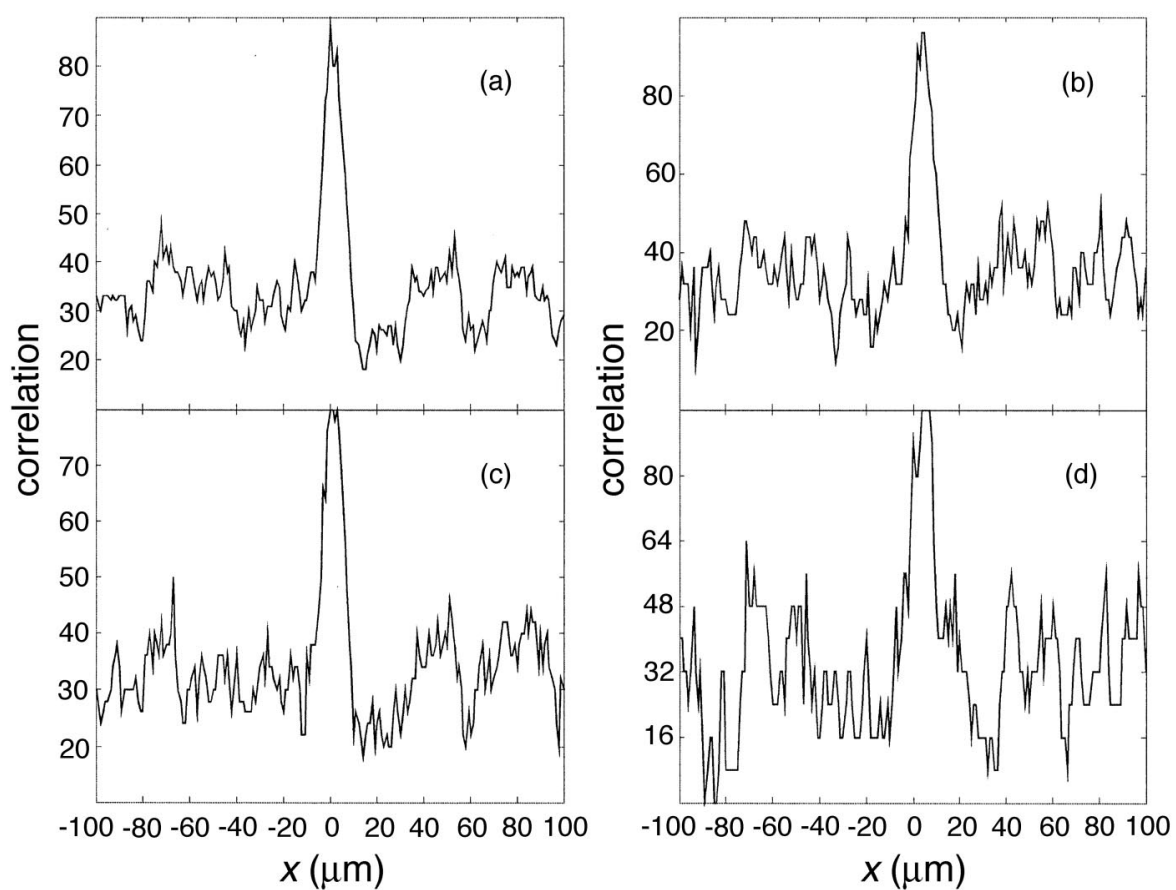

Fig. 7. Effect of the number of effective detectors in the array (binning): $4 \times 4$ binning, 128 detectors; (d) $8 \times 8$ binning, 64 detectors.

the variance of the intensity distribution. We used three binarization thresholds, given by $\langle I\rangle,\langle I\rangle+\sigma_{l} / 2$, and $\langle I\rangle+\sigma_{l}$. The results are presented in Fig 6; Figs. 6(a), 6(b), and 6(c), respectively, correspond to the thresholds just described. We also used two different correlation algorithms on the binary codes. In the first one, the signal is computed by means of the sum

$$
\sum_{i=1}^{N}\left(J_{1 i} \& J_{2 i}\right) \mid\left(\sim J_{1 i} \& \sim J_{2 i}\right),
$$

where the subscripts 1 and 2 , respectively, refer to the codes from diffusers D1 and D2 and \&, |, and stand for the operators AND, OR, and NOT, respectively. With this algorithm the coincidences of both two high values and two low values are added to the correlation sum. In the second algorithm we use the sum

$$
\sum_{i=1}^{N}\left(J_{1 i} \& J_{2 i}\right)
$$

so only the coincidences of high values (which correspond to the maxima of the speckle grains) are added to the correlation value. For each threshold, the pulses obtained with expression (4) are plotted by a solid curve. The pulses obtained with expression (5) are marked by dashed curves.

Another interesting issue is the minimum number of detectors required for good-quality reference signals. Of course, this number depends on the sizes of the speckle grains, which in turn depend on the geometry of the system, as described by Eq. (1). We changed the number of detectors by varying their size, keeping constant the length of the array. This was easily done by binning of the CCD pixels in such a way that virtual detectors were made up by cells of $1 \times 1$ to $8 \times 8$ pixels. The results are presented in Fig. 7. For Figs. 7(a), 7(b), 7(c), and 7(d) the number of detectors was $512,256,128$, and 64 , respectively. Binning levels of up to $4 \times 4$ pixels has almost no effect on the signal quality, and we found a clear increase in the noisy background level for the highest binning level. Pulse width is almost insensitive to binning.

\section{Conclusions}

A speckle-based technique for generating narrow reference pulses has been proposed and demonstrated. It may compete with actual state-of-the-art techniques with the advantage of being highly insensitive to the gap between the scale of the encoder and the reading head. Traditional strip code correlation with collimated light requires a gap tolerance of \pm 25 $\mu \mathrm{m}$ for $20-\mu \mathrm{m}$ reference pulses; $10-\mu \mathrm{m}$ pulses demand even tighter tolerances.

To improve the resolution of the method requires further reduction of the speckle grains. According to Eq. (1) this would require a shorter distance from the source to the diffuser, which is possible and convenient, as a more compact design is desirable. The results presented show that it is possible to obtain essentially the same width of a reference signal $(\approx 10$ $\mu \mathrm{m}$ ) by use of either an interference measurement or an intensity correlation measurement, although the latter is far more robust. The method based on intensity correlation is limited by the pixel size in the detection device, but the displacement amplification given by Eq. (2) can overcome this limitation. Be- 
cause of the amplification obtained with the configuration of Fig. 2, that is, four times the diffuser displacement, we got a peak approximately four times narrower than the speckle size [ $45 \mu \mathrm{m}$ in Figs. 5(a), 6, and 7]. Another advantage of this amplification factor is that larger speckle size permits larger pixels on the detectors.

Binarization of the speckle patterns has no effect on the peak width for a wide range of threshold selection. Signal degradation begins to appear only with a threshold given by the mean intensity plus the variance of the intensity distribution. The signal is also stable against variations of the number of detectors. Our setup supported a binning level of the CCD system of $4 \times 4$. These results encouraged us to develop an experimental setup with a real-time electronic correlation system.

The main novelty of this research is in the introduction of symmetrical diffusers that make the system highly insensitive to displacements of the reading head along the $z$ axis. The next step is to study the stability of the system under angular misalignments between the reading head and the diffusers. We estimate that these misalignments will cause a peak position shift, along with a decrease of the peak height in relation to the background noise, as happens in any other reference signal technology. We intend to analyze the performance of the novel design in detail in a future publication.

We thank M. Quintanilla for useful comments and discussions. This research has been supported by projects DPI 2001-1238 and DPI 2001-1369 of the Spanish Ministry for Science and Technology.

\section{References}

1. Y. Arai and T. Kurata, "A high-resolution encoder by multiplication of moire fringes," Eng. Technol. 9, 1-7 (1987).

2. K. Engelhardt and P. Seitz, "High resolution optical position encoder with large mounting tolerances," Appl. Opt. 36, 29122916 (1997).

3. Y. Wei-Hung, W. Bletscher, and M. Mansuripur, "High resolution optical shaft encoder for motor speed control based on an optical disk pick-up,” Rev. Sci. Instrum. 69, 3068-3071 (1998).

4. D. Crespo, J. Alonso, T. Morlanes, and E. Bernabeu, "Optical encoder based on the Lau effect," Opt. Eng. 39, 817-824 (2000).

5. Y. Jourlin, J. Jay, and O. Parriaux, "Compact diffractive interferometric displacement sensor in reflection," Precis. Eng. 26, 1-6 (2002).

6. X. Yang and C. Yin, "A new method for the design of zero reference marks for grating measurement systems," J. Phys. E. 19, 34-37 (1986).

7. L. Yajun, "Design of zero reference marks for grating measurement systems: a new method," Meas. Sci. Technol. 1, 848851 (1990)

8. J. W. Goodman. "Statistical properties of laser speckle patterns," in Laser Speckle and Related Phenomena, J. C. Dainty, ed., Vol. 9 of Topics in Applied Physics (Springer-Verlag, Berlin, 1984). pp. 9-75

9. R. E. Luna, E. R. Méndez, J. Q. Lu, and Z.-H. Gu, "Enhanced backscattering due to total internal reflection at a dielectricair interface," J. Mod. Opt. 42, 257-269 (1995).

10. I. Yamaguchi, "Linear and rotary encoders using electronic speckle correlation," Opt. Eng. 30, 1862-1868 (1991). 